Article

\title{
Carbon-Nanotube-Based Monolithic CMOS Platform for Electrochemical Detection of Neurotransmitter Glutamate
}

\author{
Alexandra Dudina ${ }^{1, *}$, Urs Frey ${ }^{1,2}$ (D) and Andreas Hierlemann ${ }^{1}(\mathbb{D}$ \\ 1 Bio Engineering Laboratory, Department of Biosystems Science and Engineering, ETH Zurich, \\ Mattenstrasse 26, CH-4058 Basel, Switzerland \\ 2 MaxWell Biosystems AG, CH-4058 Basel, Switzerland \\ * Correspondence: dudina.a.u@gmail.com; Tel.: +41-61-387-3314
}

Received: 4 May 2019; Accepted: 3 July 2019; Published: 12 July 2019

check for updates

\begin{abstract}
We present a monolithic biosensor platform, based on carbon-nanotube field-effect transistors (CNTFETs), for the detection of the neurotransmitter glutamate. We used an array of 9'216 CNTFET devices with 96 integrated readout and amplification channels that was realized in complementary metal-oxide semiconductor technology (CMOS). The detection principle is based on amperometry, where electrochemically active hydrogen peroxide, a product of the enzymatic reaction of the target analyte and an enzyme that was covalently bonded to the CNTFET, modulated the conductance of the CNTFET-based sensors. We assessed the performance of the CNTs as enzymatic sensors by evaluating the minimal resolvable concentration change of glutamate in aqueous solutions. The minimal resolvable concentration change amounted to $10 \mu \mathrm{M}$ of glutamate, which was one of the best values reported for CMOS-based systems so far.
\end{abstract}

Keywords: carbon nanotube field-effect transistor; CMOS; ion-sensitive field-effect transistors; ChemFET

\section{Introduction}

The integration of 1D nanomaterials, such as carbon nanotubes (CNT), into sensor array platforms yields a sensitive tool for the detection of biological species and it has significant advantages over conventional optical detection methods [1,2]. The first advantage is related to the size compatibility of sensor and analyte: the diameter of a CNT, which is $\sim 1 \mathrm{~nm}$, is comparable to the size of single molecules (e.g., the DNA molecule has a size of $1 \mathrm{~nm}$ [1]). The second advantage of CNT-based sensor platforms is that most biological processes involve electrostatic interactions and charge transfer, which can be directly detected by charge-sensitive CNTs. In the case of single-walled carbon nanotubes (SWNTs), every atom is at the surface and it is exposed to the environment and, thus, even small changes in the environment can cause significant changes in the nanotube electrical properties [3,4]. Finally, the material carbon provides a natural match to organic molecules. Thus, among different nanomaterials, carbon nanotubes have a great potential for biosensor applications.

The use of field-effect transistors, based on carbon nanotubes (CNTFETs), has been reported in literature for the electronic detection of single-molecule dynamics, [5,6], highly localized measurements of intracellular electrophysiology, [7], and for label-free detection of disease-related biomarkers and viruses $[8,9]$.

The CNTFET sensing mechanism is similar to that of ion-sensitive FETs. The charge carriers or ions in the liquid phase, located in close proximity to the CNTFETs, lead to an electrostatic potential buildup. This potential depends on the nature and concentration of the charged species and it modulates the electrical resistance of the CNTFETs [3]. 
It is known that the properties of CNTs depend strongly on their physical characteristics, such as diameter, length, the presence of residual catalyst, and their chirality [10]. For example, CNTs can be either single-walled (SWCNTs) or multi-walled (MWCNTs) with varying intrinsic bandgaps and helicities. In addition, SWCNTs can be either metallic conductors or semiconductors, based on the chirality of the structure [11].

The majority of research efforts towards bio-sensing involves interactions of proteins with CNTs. The attachment of proteins to carbon nanotubes can be performed in several steps after preliminary chemical functionalization of the CNTs. For example, carbon nanotubes can be oxidized to have free carboxyl groups that then undergo coupling with amino groups in proteins [12,13].

Although the various applications of CNTs for the detection of various bio species have been reported previously, the effects of the spread in inherent CNT characteristics, such as semiconducting versus metallic properties or the number of parallel-connected CNTs per sensor, on the device or sensor sensitivity, have yet to be established.

In this paper, we used a complementary metal-oxide semiconductor (CMOS) sensor platform, comprising an array of 9216 CNTFETs, along with readout and amplification channels, for the detection of the neurotransmitter glutamate. A dielectrophoresis-based method (DEP) was used to integrate the CNTs with the electrode array. Given the statistical nature of the DEP deposition process, a large range of CNT-sensor characteristics was observed, as there were single CNTs or bundles of parallel CNTs deposited between the electrode pairs. We characterized the CNTFET ensemble across the whole array in terms of their sensitivity to solutions of different $\mathrm{pH}$-values and showed that the sensitivity was inversely proportional to the initial baseline resistance of the CNTFETs in aqueous medium. Afterwards, we demonstrated a successful functionalization of the CNTFETs with glutamate oxidase and the detection of L-glutamate at concentrations as low as $10 \mu \mathrm{M}$ for using only one CNTFET and $3 \mu \mathrm{M}$ for averaging the signals of 62 CNTFETs. Finally, we characterized the CNTFETs across the whole array in terms of their sensitivity to solutions of L-glutamate at different concentrations and we showed the interrelation between the minimal resolvable values of glutamate concentration changes and the minimal detectable $\mathrm{pH}$-change values for the CNTFETs.

\section{Materials and Methods}

\subsection{Experimental Setup}

Figure 1 shows a schematic view of the overall system used for this work. The system comprises a monolithic CMOS chip, featuring both, the readout amplifiers and the CNTFET array. The chips were mounted on a custom-designed printed circuit board (PCB), which also provided analog reference voltages and a digital block for bidirectional commination of the chip with a data acquisition card (National Instruments, Munich, Germany).

A novel packaging for the CMOS chip was developed to protect bond wires and readout electronics whilst keeping the electrode array exposed to the liquid analyte solution. After wire-bonding of the CMOS chip to the custom-made PCB carrier, we applied an epoxy encapsulation (EPO-TEK 353, Kummer AG, Cham, Switzerland). A microfluidic chamber, made from PDMS, with a volume of approximately $10 \mu \mathrm{L}$ was mounted on top of the sensor array. A LabView (National Instruments, Munich, Germany) interface was implemented to maintain communication with the CMOS chip and to transfer the measurement data to a PC. We analyzed the acquired data with custom-made software written in Matlab (Mathworks). Each unit is described in more detail in the following sections. 


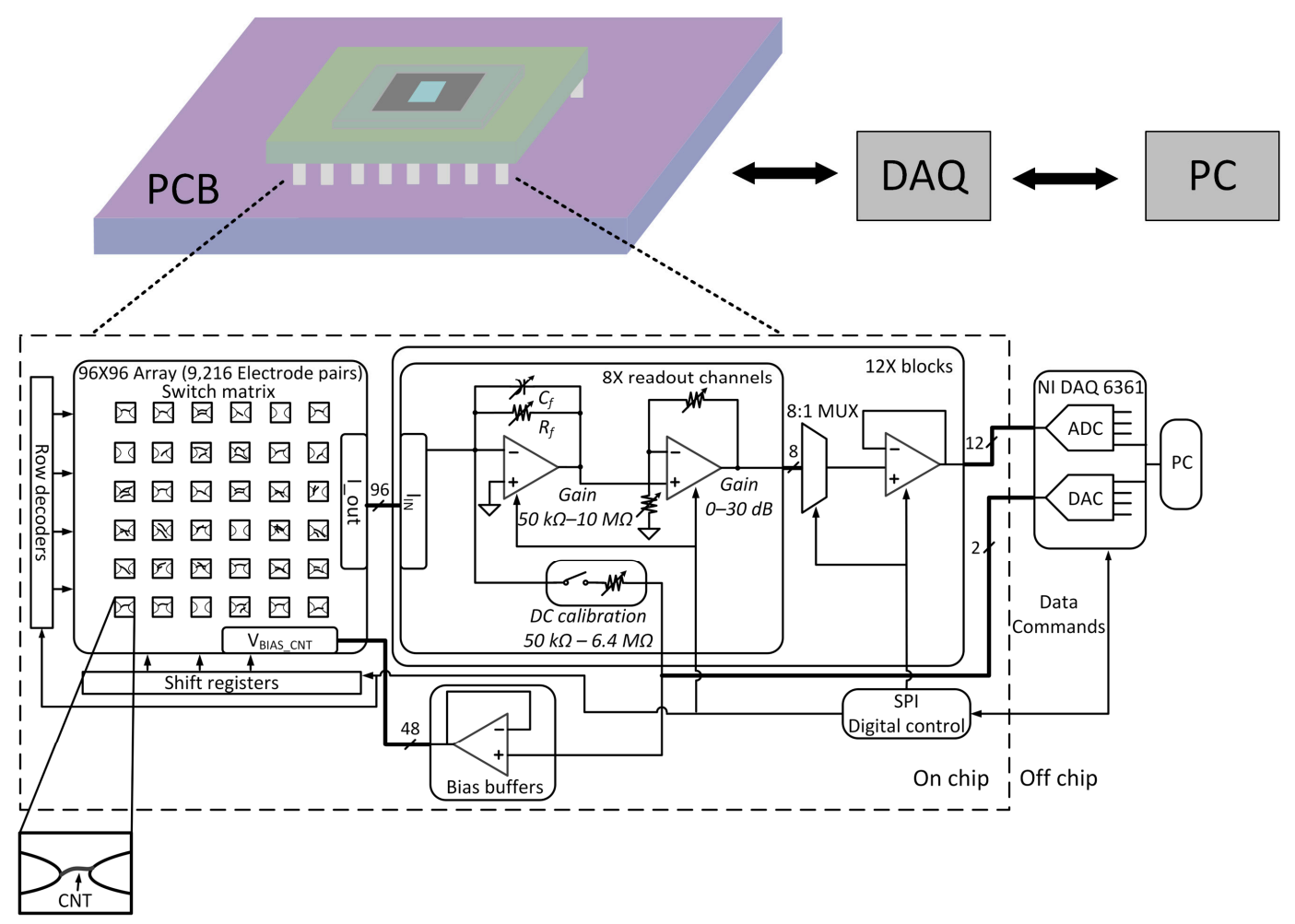

Figure 1. Block-diagram of the overall sensor system and setup (adapted from Reference [14]).

\subsection{CMOS Chip}

The CMOS readout chip included an array of 9216 platinum electrode pairs and 96 integrated readout and amplification channels. The electrode pairs provided the source and drain connection for the CNTFETs. Two adjacent drain electrodes shared one source electrode. The underlying electrode routing was switch-matrix based, similar to that described in Reference [15].

Each readout channel included two stages of amplification and filtering, followed by a multiplexer (MUX) and a driving buffer. The dedicated digital block, assigned to each readout channel, allowed for independent gain and bandwidth selection. To compensate for the offset and gain error, a resistive feedback loop was implemented in each channel. An offset voltage can add errors to the measurement of the baseline conductance of the sensor, as the bias voltage of the CNT is not precisely defined. The offset compensation was done using six different resistor values in a similar way as reported by Grassi et al in Reference [16]. To define the voltages at the source terminals of each CNTFET, the chip included 48 voltage buffers. Each voltage buffer was an operational amplifier, configured in unity-gain feedback and provided voltages in the range from $200 \mathrm{mV}$ to up to $3.2 \mathrm{~V}$.

The CMOS chip was fabricated in $0.18-\mu \mathrm{m}-\mathrm{CMOS}$ technology, and the overall chip real estate was $6.4 \times 3.0 \mathrm{~mm}^{2}$. The system was successfully tested and it showed a noise performance of $2.14 \mathrm{pArms}$ at a bandwidth of $1 \mathrm{kHz}$, and $0.84 \mathrm{nArms}$ at a bandwidth of $1 \mathrm{MHz}$. Resistances in a range between $50 \mathrm{k} \Omega$ and $1 \mathrm{G} \Omega$ could be resolved. The chip die is shown in Figure 2.

\subsection{Fabrication and CNT Assembly}

Pairs of platinum electrodes were patterned at wafer level by means of ion-beam metal deposition and etching. A SEM picture of the fabricated Pt electrodes is shown in Figure 2.

To achieve a covalent attachment of proteins onto the sidewalls of CNTs, we used single-walled CNTs (SWCNTs) with carboxylic-acid functionalization (Sigma Aldrich, Buch, Switzerland). These nano-devices had a length of approximately $3 \mu \mathrm{m}$ and a diameter of approximately $2 \mathrm{~nm}$. The CNTs were assembled on the CMOS chip by means of a dielectrophoresis (DEP)-based deposition technique. The details of the assembly process were described in References $[14,17]$ and were only 
briefly abstracted in this paper. The initial suspension was prepared by mixing dry CNT powder with distilled MilliQ water, and the mixture was subsequently sonicated for $30 \mathrm{~min}$ to obtain a homogeneous suspension. The final concentration of the suspension was approximately $80 \mu \mathrm{g} / \mathrm{L}$. A wire-bonded, packaged CMOS chip was mounted onto the setup board. The switch-matrix below the electrodes was configured to connect all the electrode pairs in the array to two defined pads of the CMOS chip [14]. An external AC source providing a voltage amplitude of $4 \mathrm{~V}_{\mathrm{pk} \text {-pk }}$ at a frequency of $600 \mathrm{kHz}$ was connected through the pads to the electrode pairs to create an electrical field. The assembly process was carried out for one hour, thereafter the chip was rinsed with deionized water to wash out the rest of the suspension, and it was then gently dried with nitrogen. In Figure 2, one electrode pair with the assembled CNTs is shown.

Figure 3a represents a physical mapping of the whole array featuring CNTs bridging the electrode pairs. The measurements have been performed in dry air with a bias voltage, $\mathrm{V}_{\mathrm{ds}}$, of $100 \mathrm{mV}$. Figure $3 \mathrm{~b}$ shows the histogram of the corresponding resistances. Owing to imperfections of the packaging process, the boundary region at the top was covered with epoxy and, therefore, it was not available for the assembly process.

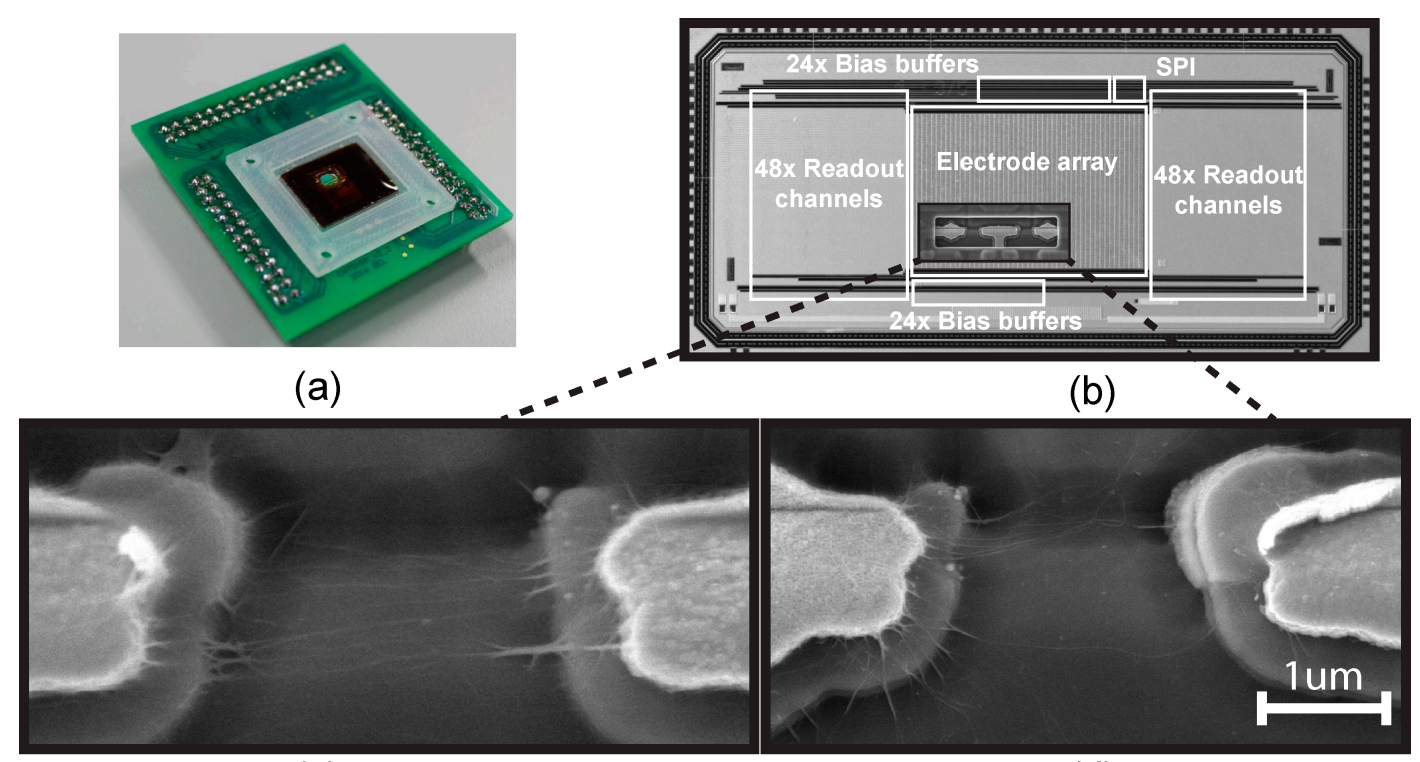

(c)

(d)

Figure 2. (a) Packaged chip; (b) Micrograph of a chip die with an area of $6.4 \times 3.0 \mathrm{~mm}^{2}$; (c,d) Scanning Electron Microscopy (SEM) pictures showing electrode pairs with integrated carbon nanotubes (CNTs).

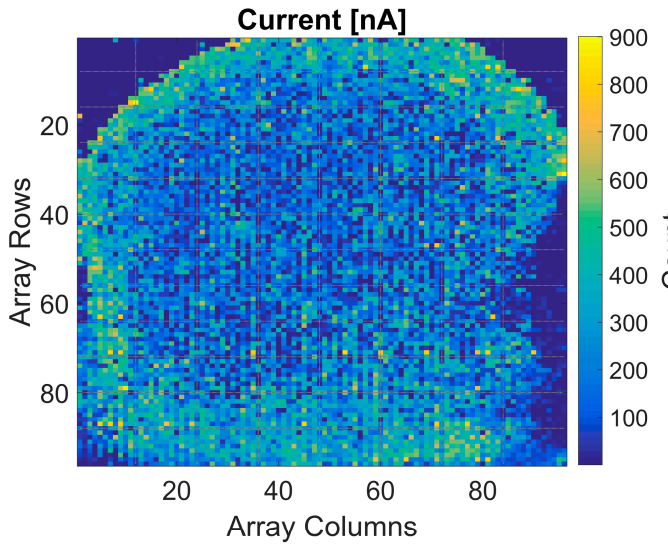

(a)

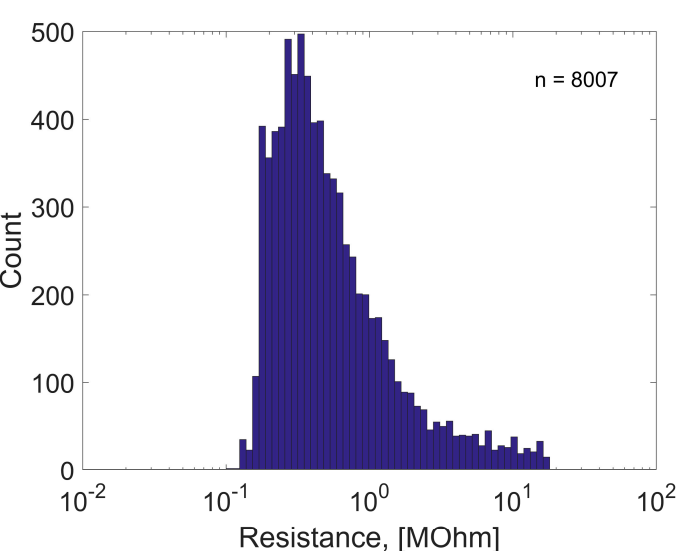

(b)

Figure 3. (a) Physical map of 8007 CNT-bridged electrode pairs as obtained from electrical conductance tests, (b) Histogram of resistance values across the whole array. 


\subsection{Functionalization Protocol}

All chemicals were purchased from Sigma Aldrich, Buchs, Switzerland. They included 1-ethyl-3-(3-dimethylaminopropyl) carbodiimide (EDC), N-hydroxysulfo-succinimide (sulfo-NHS), L-glutamate oxidase from Streptomyces, Tris HCL, Bovine Serum Albumine (BSA), and l-glutamic acid. The CNT functionalization procedure was adapted from Reference [13] and it is briefly illustrated in Figure 4. A CNT array was immersed in a freshly prepared 3-mL aqueous solution of EDC $(10 \mathrm{mg} / \mathrm{mL})$, see Figure $4 \mathrm{a}$. Under stirring, $90 \mathrm{mg}$ of sulfo-NHS was added to the solution. The reaction was allowed to proceed at room temperature for $2 \mathrm{~h}$, Figure $4 \mathrm{~b}$. Glutamate oxidase (GlOx) was dissolved at a concentration of $2 \mathrm{mg} / \mathrm{mL}$ in a filter-sterilized phosphate buffer solution (PBS) with a $\mathrm{pH}$ of 8. The volume of the prepared sample was $3 \mathrm{~mL}$. An array chip was washed quickly with MilliQ water and immediately immersed into the prepared solution. The enzyme immobilization reaction occurred at room temperature over $1 \mathrm{~h}$, Figure 4c. After that, the electrode surface was washed with a phosphate buffer solution ( $\mathrm{pH} 7.4$ ) and further incubated for 30 mins in $0.1 \mathrm{M}$ Tris $\mathrm{HCl}$ in PBS. The CNTFET surface was washed with PBS and then, the electrodes were blocked with $0.5 \%$ BSA in PBS of pH 7.4 for $1 \mathrm{~h}$. Finally, the CNTFET surface was washed one more time in PBS and prepared for the biological experiments.

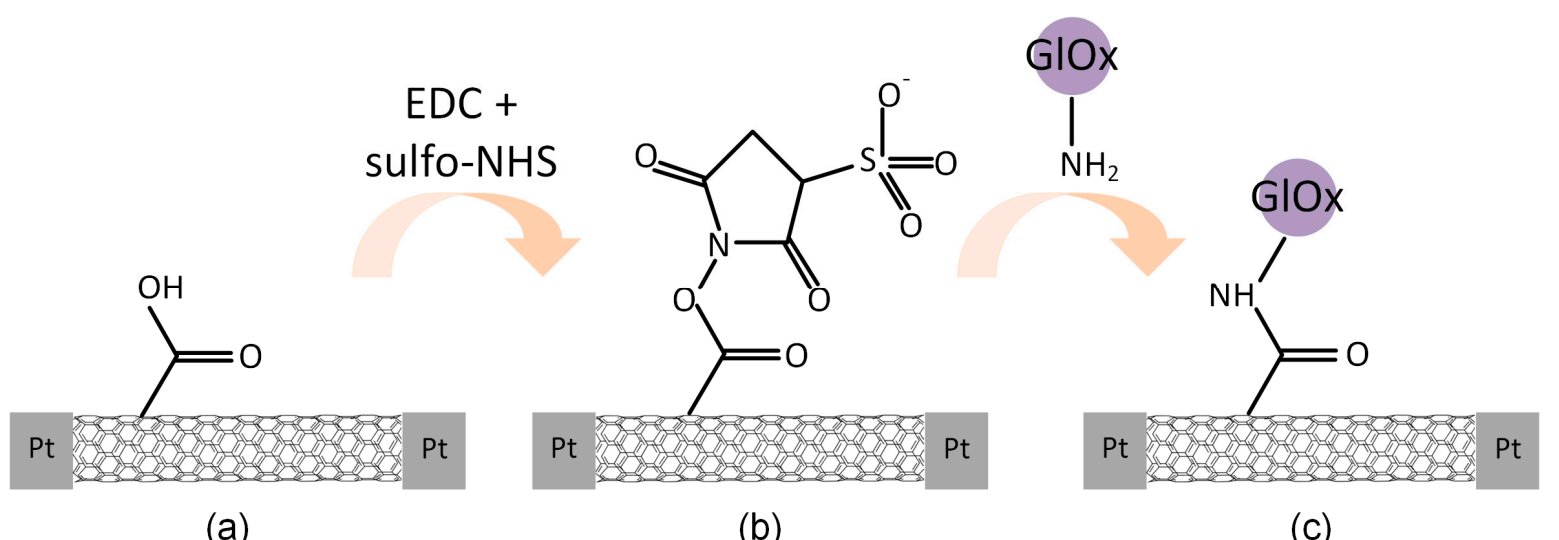

Figure 4. Illustration of the functionalization steps with the corresponding enzymes: (a) CNTs featuring carboxylic groups at the surface; (b) intermediate stage after the EDC and sulfo-NHS coupling; (c) final state after coupling with glutamate oxidase.

\section{Results and Discussion}

\subsection{Sensor Characterization}

First, the current-voltage (IV) curves of the CNTFET sensors connecting the electrode pairs were recorded. The sensor characterization was performed before the attachment of enzyme, i.e., the CNTs only had carboxylic groups on the surface. The measurements were performed in a phosphate-buffered saline (PBS, pH = 7.4), (Gibco, Life Technologies, Zug, Switzerland), diluted ten-fold in MilliQ water (10 mM PBS). Using diluted PBS, the ionic strength of the liquid solution could be reduced, which increased the Debye screening length (i.e., the distance at which the surface potential of the CNTFET sensor was completely screened, which resulted in zero response to analytes in solution) [18]. The source-drain voltage was set to $100 \mathrm{mV}$, whilst the electrolytic gate potential was swept from $-400 \mathrm{mV}$ to $250 \mathrm{mV}$ with respect to the source potential.

The values of the drain current at a gate potential of $-400 \mathrm{mV}$ and $250 \mathrm{mV}$ were defined as $\mathrm{I}_{\mathrm{ON}}$ and $\mathrm{I}_{\mathrm{OFF}}$. The value of the CNTFET resistance at a gate potential of $-400 \mathrm{mV}$ was defined as $R_{\mathrm{ON}}$. Figure 5 a shows the plot of the ratio of $\mathrm{I}_{\mathrm{ON}} / \mathrm{I}_{\mathrm{OFF}}$ values of each CNTFET device versus $\mathrm{R}_{\mathrm{ON}}$ over the full array. These values can be used to deduce the nature of the CNT devices (semiconducting or metallic). The devices featuring higher resistances were considered to be semiconducting CNTs and 
show a higher $\mathrm{I}_{\mathrm{ON}} / \mathrm{I}_{\mathrm{OFF}}$ ratio, whereas the devices with lower resistance were considered to be metallic or bundles of CNTs of mixed type.

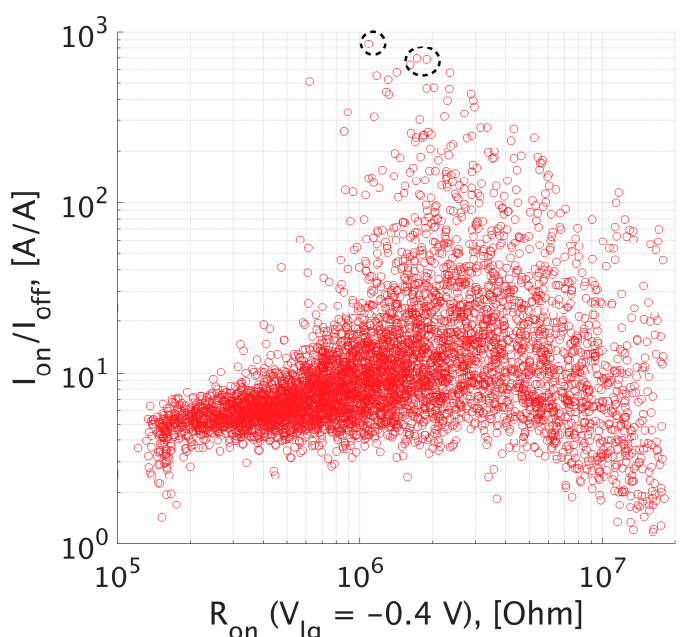

(a)

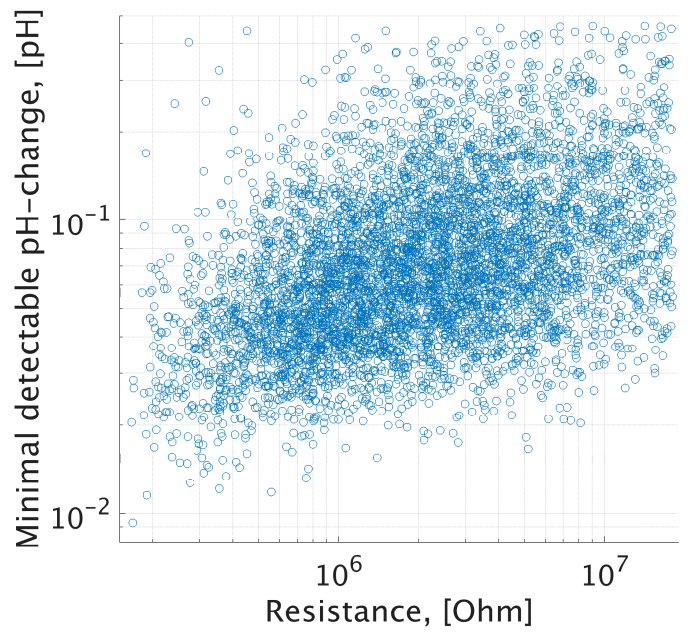

(c)

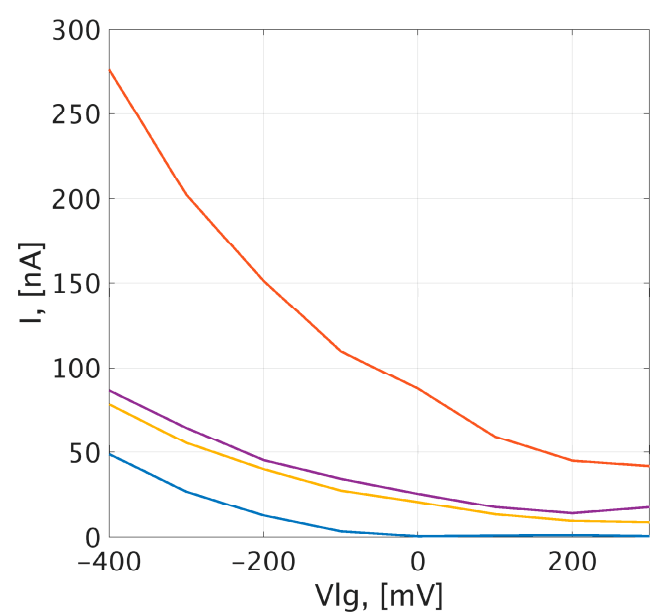

(b)

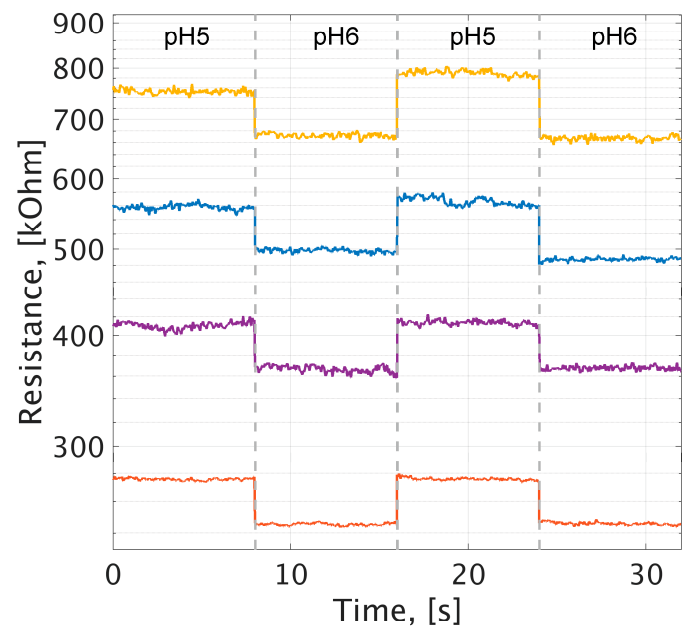

(d)

Figure 5. (a) Scatter plot of the $\mathrm{I}_{\mathrm{ON}} / \mathrm{I}_{\mathrm{OFF}}$ ratio versus the $\mathrm{R}_{\mathrm{ON}}$ of all carbon nanotube field-effect transistors (CNTFET) devices of an array. (b) Current-voltage characterization (I-V curves) of CNTFETs with the largest $\mathrm{I}_{\mathrm{ON}} / \mathrm{I}_{\mathrm{OFF}}$. (c) Scatter plot of minimal detectable $\mathrm{pH}$-changes versus $\mathrm{R}_{\mathrm{PH} 5}$; (d) Resistance changes versus time of 4 different CNTFET devices upon sweeping the $\mathrm{pH}$ values from 5 to 6 two times.

Figure $5 \mathrm{~b}$ shows the I-V curves of CNTFETs featuring the highest $\mathrm{I}_{\mathrm{ON}} / \mathrm{I}_{\mathrm{OFF}}$ ratio, marked by the dashed circle. The CNTFETs appeared to behave like p-type field-effect transistors, exhibiting an overall electrical conductance decrease upon sweeping the potential of the reference electrode from negative to positive values with respect to the source potential.

We performed a CNTFET sensitivity analysis, namely the estimation of the minimal detectable $\mathrm{pH}$-change value across all CNTFETs over the whole array. Sodium phosphate buffer solutions with two different $\mathrm{pH}$-values were prepared. The solutions of $\mathrm{pH}$-values 5 and 6 were applied sequentially to the microfluidic chamber hosting the CNTFET array. $\mathrm{V}_{\mathrm{ds}}$ was set to $100 \mathrm{mV}$, the electrolytic gate potential was set to $-300 \mathrm{mV}$. The measured data were recorded by simultaneously sampling 96 -channels at $4 \mathrm{kHz}$ and then sequentially scanning the whole CNTFET array. The response of every CNTFET sensor was recorded over $8 \mathrm{~s}$.

The acquired raw data were low-pass filtered with a corner frequency of $100 \mathrm{~Hz}$. The estimation of the minimal resolvable $\mathrm{pH}$-change value was based on a calculation of the Fisher information (statistics). The Fisher information describes the variance of the mean value of the measured experimental dataset. 
Finally, the resolution was defined, based on the Cramer Rao bound, which is the inverse of the square root of the Fisher information. Figure $5 \mathrm{c}$ shows the scatter plot of the minimal detectable $\mathrm{pH}$-change value of individual CNTFETs at $\mathrm{pH}=5, \mathrm{R}_{\mathrm{pH}}$, is shown versus their baseline resistance. The best-achieved value was as low as $0.01 \mathrm{pH}$ units for individual CNTFETs. From the figure, the sensitivity of the CNTFETs was inversely proportional to the initial resistance of the CNTFETs, so that the sensitivity of devices with lower resistance was higher than the devices featuring higher resistance. This finding could be explained by the fact that the $\mathrm{pH}$ sensitivity was based on the protonation or deprotonation of the functional groups at the surface of the CNTFETs. At lower $\mathrm{pH}$ values, ( $\mathrm{pH} 5)$, the carboxylic groups get protonated, which provides positive charges on the gate, whereas, at higher $\mathrm{pH}$ values, the carboxylic groups get deprotonated, which provides negative charges on the gate. The slope of the I-V curve, however, depends on the formation of a double layer and the accumulation of positive $\left(\mathrm{Na}^{+}\right)$or negative $\left(\mathrm{Cl}^{-}\right)$charges at the surface of the CNTFET devices, which depends on the potential that has been set at the reference electrode. It is likely that CNTFETs featuring lower resistance values feature bundles of CNTs that are connected in parallel between the electrode pair. A bundle of CNTs has more functional groups at the CNTFET surface, which contribute to the change of the sensor resistance upon changing the solution $\mathrm{pH}$. Figure $5 \mathrm{~d}$ shows the $\mathrm{pH}$ traces of four individual CNTFETs versus time. The minimal resolvable $\mathrm{pH}$ change for these sensors amounted to approximately $0.01 \mathrm{pH}$-units. The CNTFETs showed a good stability and a very small drift.

\subsection{Detection of Neurotransmitters}

Glutamate is an excitatory neurotransmitter, which is released at synaptic terminals [19]. It plays a major role in various neural functions and relates to various neurological disorders, as well as to memory and learning processes [20]. For example, an excessive release of glutamate plays a key role in neuronal death associated with a wide range of neural disorders [20]. Therefore, real-time monitoring of extracellular glutamate levels would be very helpful in understanding the excitotoxic process of neurotransmitters in the case of brain injury.

Biosensors for glutamate are usually based on glutamate oxidase (GlOx). The working principle is illustrated in Figure 6. The GlOx catalyzes the oxidation of glutamate to 2-oxoglutarate in the presence of oxygen, which produces electrochemically active $\mathrm{H}_{2} \mathrm{O}_{2}$.

$$
\text { Glutamate }+\mathrm{O}_{2} \rightarrow \alpha \text {-ketoglutarate }+\mathrm{NH}_{3}+\mathrm{H}_{2} \mathrm{O}_{2}
$$

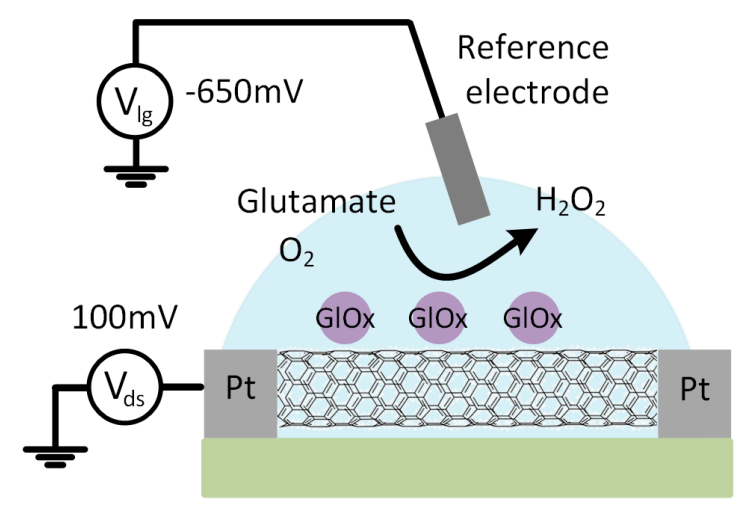

Figure 6. Illustration of the working principle of enzyme-functionalized CNT-based biosensors.

Quantification of glutamate by means of amperometric biosensors is achieved via electrochemical oxidation of the liberated $\mathrm{H}_{2} \mathrm{O}_{2}[19,21]$. The oxidation of $\mathrm{H}_{2} \mathrm{O}_{2}$ requires application of a potential of $-0.65 \mathrm{~V}$ to the reference electrode. The concentrations of glutamate in extracellular recordings in neuronal preparations are in the range between a few $\mu \mathrm{M}$ to up to few tens of $\mu \mathrm{M}$ [19]. 
All solutions were freshly prepared and kept at room temperature. All experiments were carried out at room temperature. The enzymes were attached to the carboxylic functional groups of the CNTs using standard protocol of EDC and sulfo-NHS coupling, namely, forming amide linkages between the enzyme amine residues and the carboxylic-acid groups of the CNTFETs [12]. The functionalization protocol has been described in the Materials and Methods section.

Figure 7a shows the I-V curves of one randomly selected CNTFET, measured before and after functionalization. The measurements were done in PBS $(10 \mathrm{mM})$. During the measurements, the bias voltage of the CNTFET was set to $100 \mathrm{mV}$, whereas the potential at the reference electrode was swept from $-400 \mathrm{mV}$ to $200 \mathrm{mV}$. We observed a decrease in conductance of the CNTFETs upon GlOx attachment, which could be explained by the effect of a positive charge introduced to the CNTFET surface $[22,23]$. An adsorption of a positively charged species induces additional negative charges in the CNTFET, thus n-doping of the CNT and a shifting of the I-Vlg curve towards more negative gate voltage [3]. Figure $7 \mathrm{~b}$ shows the power spectral density (PSD) of the CNTFET before and after functionalization. During the noise measurements, the potential of the reference electrode was set to $0 \mathrm{~V}$. The enzyme, attached to the surface of CNTFET, introduced additional noise, mostly in the low-frequency range [24].

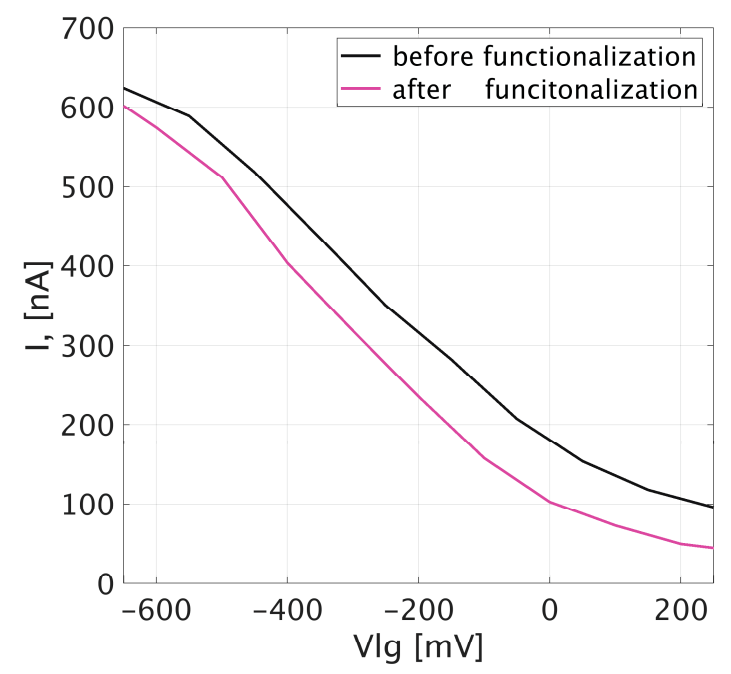

(a)

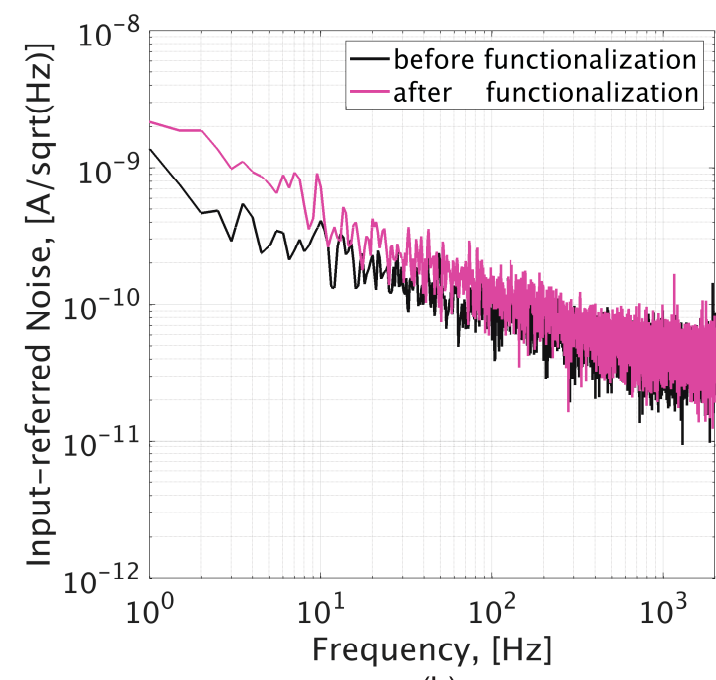

(b)

Figure 7. (a) Current-voltage characterization (I-Vlg curve) of a CNTFET before (green) and after (red) enzyme immobilization; (b) Input-referred power spectral density (PSD) of the corresponding CNTFET device, recorded in phosphate-buffered saline (10 mM PBS).

L-glutamic acid was dissolved in $10 \mathrm{mM}$ PBS at different concentrations. The solutions were freshly prepared and kept at room temperature during the experiments. The amperometric response to glutamate for two concentrations of $250 \mu \mathrm{M}$ and $500 \mu \mathrm{M}$ was recorded under steady-state conditions by applying a potential of $-650 \mathrm{mV}$ to the $\mathrm{Ag} / \mathrm{AgCl}$ reference electrode. The CNTFETs were biased at $100 \mathrm{mV}$. We defined the resistance value, $R_{P B S}$, at $10 \mathrm{mM}$ PBS as the reference value. In Figure 8a, the absolute ratio of current change, $\Delta \mathrm{I}=\mathrm{I}_{250 \mu \mathrm{Mo}}-\mathrm{I}_{\mathrm{PBS}}$, is plotted versus the respective resistance values, $\mathrm{R}_{\mathrm{PBS}}$. We observed that the current changes of the CNTFET devices upon an increase in the concentration of the analyte solution depended on the initial resistance of the CNTFET devices, and that the $\triangle \mathrm{I}$-ratio values ranged between $48 \mathrm{nA}$ and approximately $160 \mathrm{pA}$. The CNTFET devices with lower initial resistance exhibited higher absolute current changes. A hypothesis to explain this finding was that the sensitivity depended on the number of functional groups to which the enzyme was attached. CNTFETs with lower resistance values were comprised of bundles of CNTs, which feature more functional groups, so that more enzyme molecules could be attached to those groups and were available for the analyte-detection reaction. Figure $8 \mathrm{~b}$ shows the distribution of the relative CNTFET 
current change ratios, $\mathrm{I}_{250 \mu \mathrm{Mo}} / \mathrm{I}_{\mathrm{PBS}}$. The average value amounted to 1.07. The amperometric responses of six different CNTFETs upon sweeping the concentration of glutamate is presented in Figure 8c. The sensors showed a linear behavior for the measured concentrations of 250 and $500 \mu \mathrm{M}$. The response time of the individual CNTFETs to a change in the solution concentration was approximately $4 \mathrm{~s}$. A scatter plot of minimally resolvable glutamate concentration changes versus initial sensor resistance, $R_{P B S}$, is shown in Figure $8 d$. The estimation of the values was performed in a similar way as in the case of the minimal resolvable changes in $\mathrm{pH}$-values by calculation of the Fisher information and the Cramer Rao bound as the inverse of the square root of the Fisher information. From the plot, the CNTFETs with lower initial resistance showed higher sensitivity. The best achieved value was $10 \mu \mathrm{M}$ of glutamate concentration change.

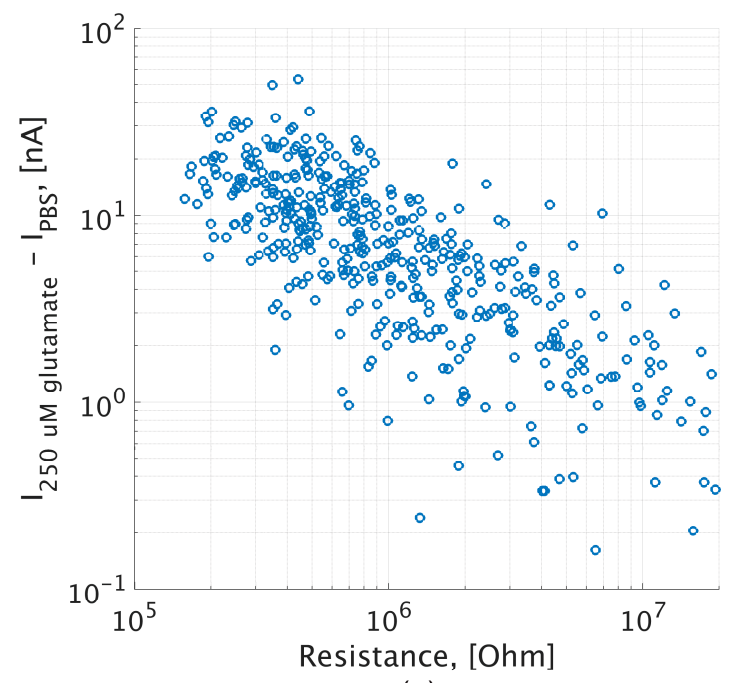

(a)

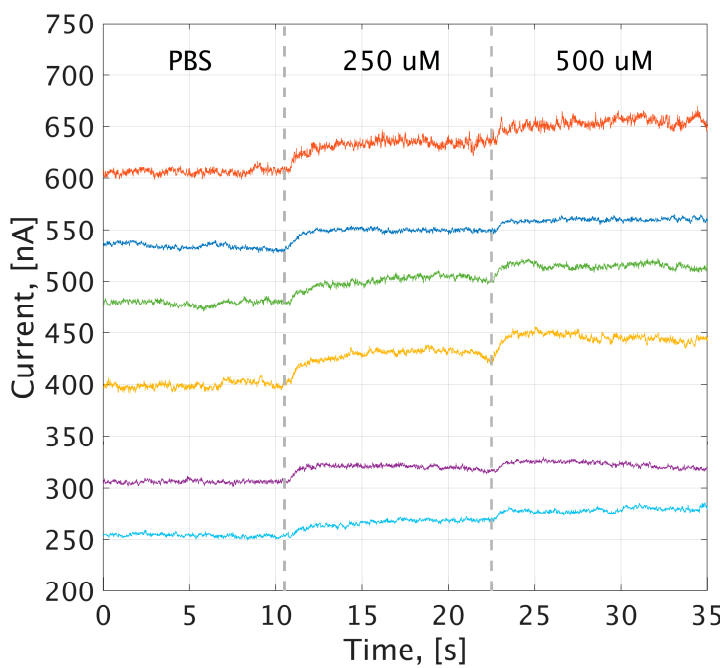

(c)

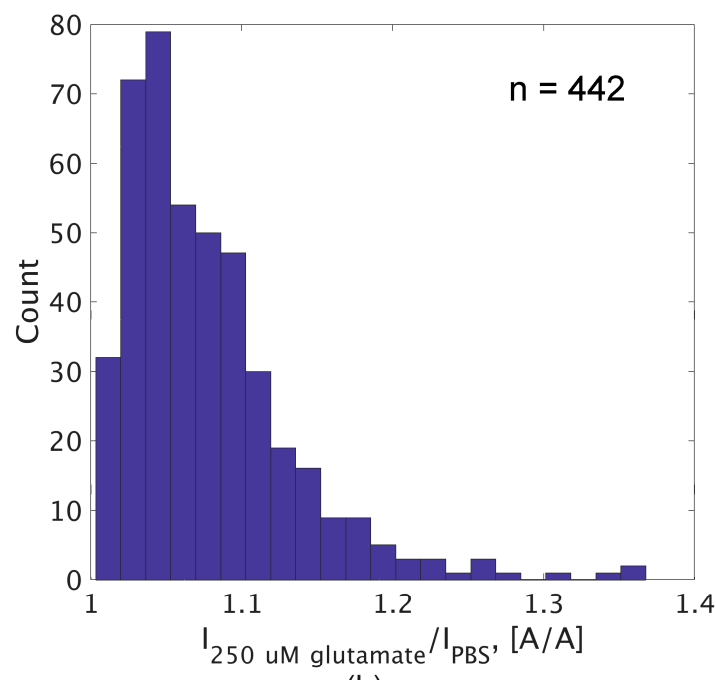

(b)

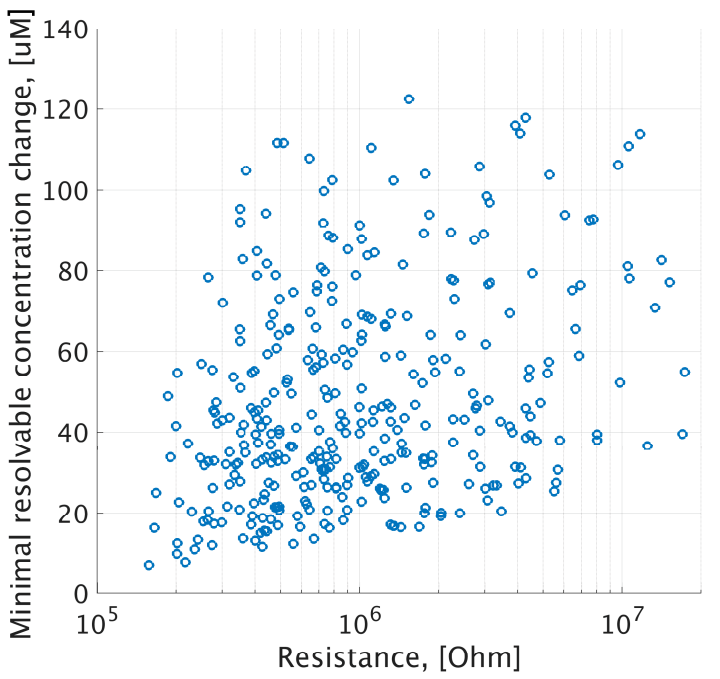

(d)

Figure 8. (a) Scatter plot of the absolute changes of the CNTFET currents, $\Delta I=I_{250 \mu \mathrm{M} \_ \text {glutamate }}-\mathrm{I}_{\mathrm{PBS}}$ versus the respective initial resistance values, $\mathrm{R}_{\mathrm{PBS}}$; (b) Histogram of the respective relative current changes of 442 CNTFETs of the array; (c) Amperometric curves versus time of 6 different individual CNTFET devices upon applying glutamate solution of two different concentrations; (d) Scatter plot of the minimal resolvable values of glutamate concentration changes versus the respective initial resistance values, $\mathrm{R}_{\mathrm{PBS}}$.

We also studied how the minimal resolvable concentration change values scale with the number of used CNTFETs. In Figure 9a, we plot the scaling of the minimal resolvable L-glutamate concentration 
change value with the number of used CNTFET sensors from one randomly selected block. The best achieved value was $3 \mu \mathrm{M}$ of glutamate upon averaging 62 CNTFETs out of 96 in one randomly recorded block. The other 34 CNTFETs in this block did not show any response, or there was no CNT connecting the electrode pair. The averaging of the signals of 62 CNTFETs gave approximately a 5.6 times relative improvement of the minimal resolvable concentration change value. We observed a similar trend in the measurement data recorded from another randomly selected block.

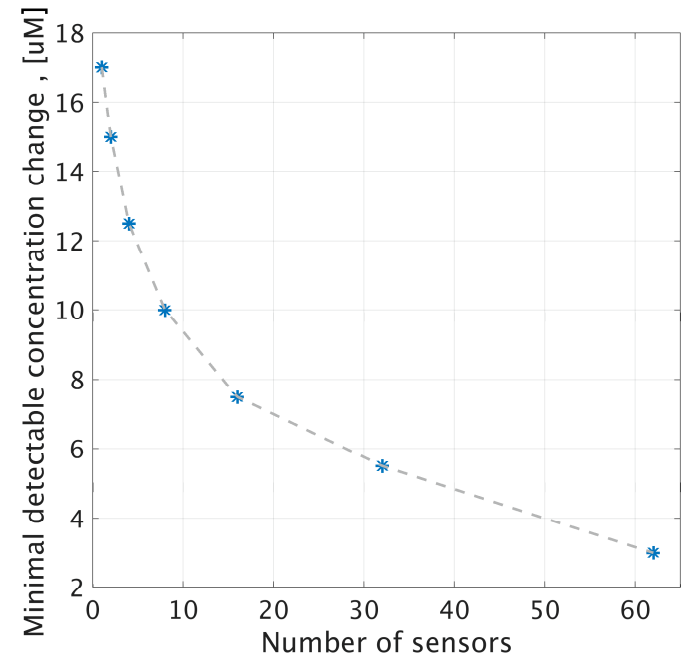

(a)

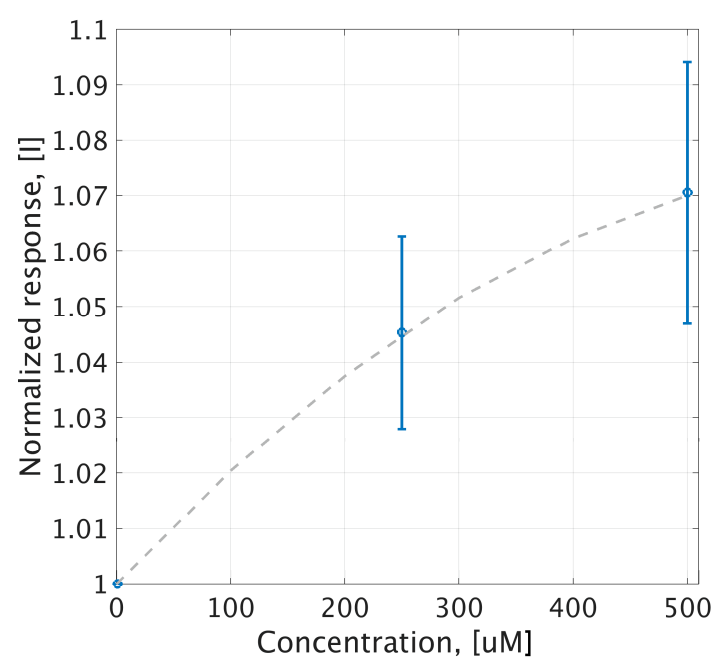

(b)

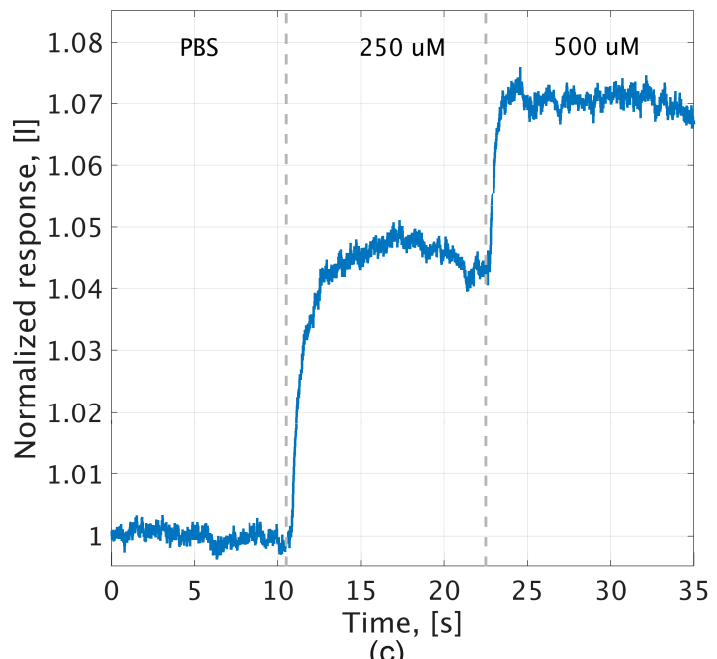

(c)

Figure 9. (a) Scaling of the minimal resolvable L-glutamate concentration with the number of used CNTFET devices; (b) Calibration curve of 62 devices. The relative non-linearity amounted to 3\%; (c) Normalized, averaged current response, [I], of 62 CNTFET devices upon applying L-glutamate solutions of different concentrations.

The calibration curve of the averaged normalized current responses of 62 CNTFETs over the concentration range between $250 \mu \mathrm{M}$ and $500 \mu \mathrm{M}$ is shown in Figure $9 \mathrm{~b}$. The curve shows an approximately quadratic dependence on the analyte concentration. Figure $9 \mathrm{c}$ shows the averaged normalized amperometric curve (normalization to the initial current, when only PBS solution has been applied to the CNTFET sensor array) of 62 CNTFET devices for the two applied analyte concentrations.

Table 1 presents a comparison of the performance of the designed system with systems reported in other references. 
Table 1. Comparison of the performance of the designed system with the other references.

\begin{tabular}{cccc}
\hline Parameter & {$[19]$} & {$[25]$} & This work \\
\hline System & Passive electrodes & CMOS chip & CMOS chip \\
\hline Number of CNT sensors & 1 & 64 & $9^{\prime} 216$ \\
\hline Number of channels & 1 & 1 & 96 \\
\hline Limit of detection & $5 \mu \mathrm{M}$ & $10 \mu \mathrm{M}$ & $10 \mu \mathrm{M}(3 \mu \mathrm{M}$ for 62 CNTFETs $)$ \\
\hline
\end{tabular}

A scatter plot of minimally resolvable glutamate concentration changes versus the minimal detectable $\mathrm{pH}$-changes value is shown in Figure 10. From the figure, the CNTFETs featuring better sensitivity to $\mathrm{pH}$-change values show a higher sensitivity to glutamate concentration changes. This finding could also be explained by the fact that the $\mathrm{pH}$ sensitivity, as well as the detection of the enzyme, is based on the chemical reaction of the functional carboxylic groups on the CNTFETs surface either with ions in the solution or with the target enzyme. The method to characterize the sensitivity of CNTFETs with different $\mathrm{pH}$ buffer solution may potentially be applied to estimate the sensitivity and the performance of the fabricated sensor array featuring the target enzyme or to fabricate the sensor arrays with graded sensitivity.

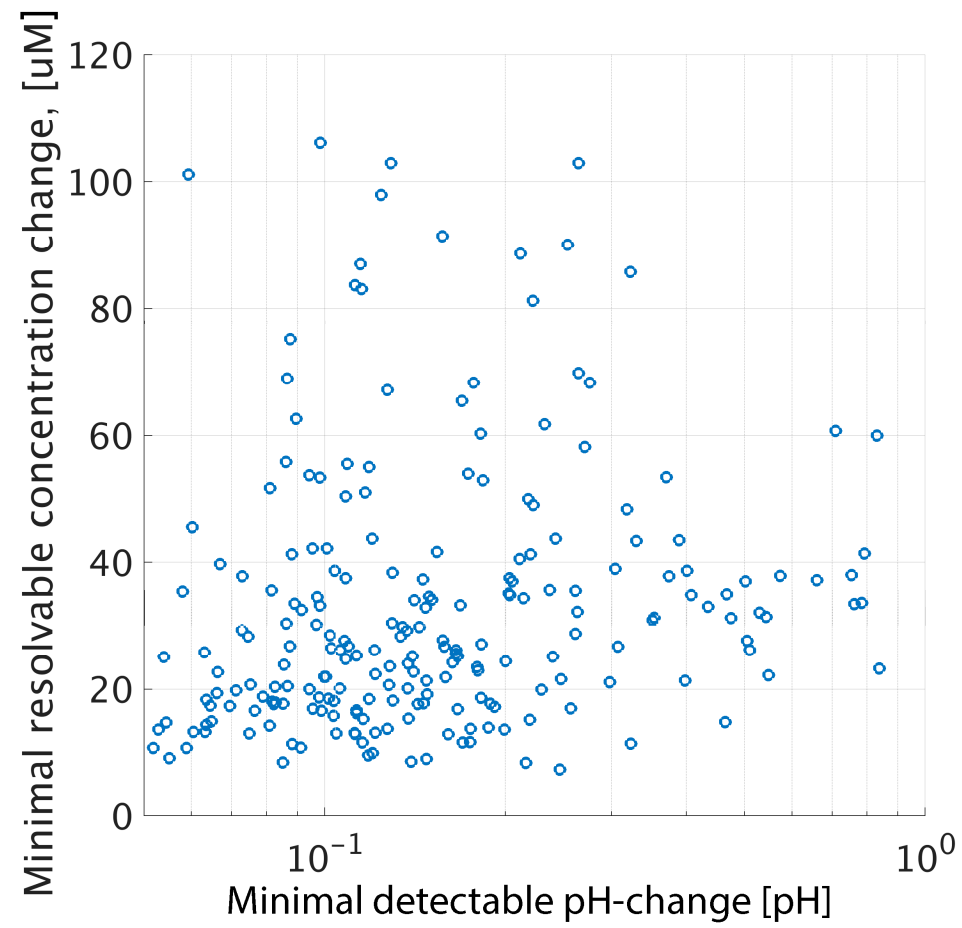

Figure 10. Scatter plot of minimal resolvable values of glutamate concentration changes versus the minimal detectable $\mathrm{pH}$-change values.4. Conclusions

We presented a monolithic CMOS system for electrochemical detection of the neurotransmitter glutamate. The system features a large array of CNTFETs, as well as 96 tunable readout and amplification channels. The system sensing functionality was verified through the measurement of CNTFET responses to buffer solutions of different $\mathrm{pH}$ values. The performance of the CNTFETs as $\mathrm{pH}$ sensors was assessed by determining the minimal resolvable $\mathrm{pH}$-change. The best achieved value was as low as $0.01 \mathrm{pH}$ units. It was demonstrated that the sensitivity of the CNTFETs was inversely proportional to their initial baseline resistance. A successful functionalization of the CNTFETs with the enzyme glutamate oxidase and the detection of L-glutamate was demonstrated. The performance of the CNTFETs as enzymatic biosensors was assessed by evaluating the minimal resolvable concentration 
change of the corresponding analyte. The system was able to detect concentrations within the physiological range. The best achieved values amounted to $\sim 10 \mu \mathrm{M}$ of L-glutamate for individual CNTFETs, whereas for several averaged CNTFETs, this value was as low as $3 \mu \mathrm{M}$ of L-glutamate.

The achieved results demonstrate the potential of CNT-based monolithic sensor platforms. The variation and control of the parameters of the assembly process will provide the potential to fabricate sensor arrays with graded sensitivity, which can be useful for various biomedical applications.

Author Contributions: A.D., U.F. and A.H. planned the conceptualization, methodology and experiments; A.D. performed the experiments and data analysis; A.D. prepared the original draft of the manuscript. All authors reviewed and edited the manuscript.

Funding: Financial support through the European Union ERC Advanced Grants "neuroXscales", under contract number AdG 694829 (H2020) is acknowledged. The funders had no role in the design of the study; in the collection, analyses, or interpretation of data; in the writing of the manuscript, or in the decision to publish the results.

Acknowledgments: We acknowledge the ZMB (Zentrum für Mikroskopie) of the University of Basel, Switzerland, for taking the SEM pictures, Peter Rimpf, ETH, for bonding the CMOS chips, Florent Seichepine, Imperial College London, Hamlyn Centre, Great Britain and Jamal Daoud, McGill University, Department of Biomedical Engineering, Canada, for useful discussions regarding the functionalization protocol.

Conflicts of Interest: U.F. is a co-founder of MaxWell Biosystems AG, Mattenstrasse 26, Basel, Switzerland. All other authors declare no conflict of interest.

\section{References}

1. Allen, B.L.; Kichambare, P.D.; Star, A. Carbon nanotube field-effect-transistor-based biosensors. Adv. Mater. 2007, 19, 1439-1451. [CrossRef]

2. Qi, P.; Vermesh, O.; Grecu, M.; Javey, A.; Wang, Q.; Dai, H.; Peng, S.; Cho, K.J. Toward large arrays of multiplex functionalized carbon nanotube sensors for highly sensitive and selective molecular detection. Nano Lett. 2003, 3, 347-351. [CrossRef]

3. Heller, I.; Janssens, A.M.; Männik, J.; Minot, E.D.; Lemay, S.G.; Dekker, C. Identifying the mechanism of biosensing with carbon nanotube transistors. Nano Lett. 2008, 8, 591-595. [CrossRef] [PubMed]

4. Bradley, K.; Briman, M.; Star, A.; Gruner, G. Charge Transfer from Adsorbed Proteins. Nano Lett. 2004, 4, 253-256. [CrossRef]

5. Choi, Y.; Moody, I.S.; Sims, P.C.; Hunt, S.R.; Corso, B.L.; Perez, I.; Weiss, G.A.; Collins, P.G. Single-Molecule Lysozyme Dynamics Monitored by an Electronic Circuit. Science 2012, 335, 319-324. [CrossRef] [PubMed]

6. Sorgenfrei, S.; Chiu, C.; Gonzalez, R.L.; Yu, Y.-J.; Kim, P.; Nuckolls, C.; Shepard, K.L. Label-free single-molecule detection of DNA-hybridization kinetics with a carbon nanotube field-effect transistor. Nat. Nanotechnol. 2011, 6, 126-132. [CrossRef] [PubMed]

7. Schrlau, M.G.; Dun, N.J.; Bau, H.H. Cell electrophysiology with carbon nanopipettes. ACS Nano 2009, 3, 563-568. [CrossRef] [PubMed]

8. Münzer, A.M.; Michael, Z.P.; Star, A. Carbon nanotubes for the label-free detection of biomarkers. ACS Nano 2013, 7, 7448-7453. [CrossRef] [PubMed]

9. Gruner, G. Carbon nanotube transistors for biosensing applications. Anal. Bioanal. Chem. 2006, 384, 322-335. [CrossRef]

10. Saifuddin, N.; Raziah, A.Z.; Junizah, A.R. Carbon Nanotubes: A Review on Structure and Their Interaction with Proteins. J. Chem. 2013, 2013, 676815. [CrossRef]

11. Dai, H. Carbon nanotubes: Synthesis, integration, and properties. Acc. Chem. Res. 2002, 35, 1035-1044. [CrossRef] [PubMed]

12. Gao, Y.; Kyratzis, I. Covalent Immobilization of Proteins on Carbon Nanotubes Using the Cross-Linker 1-Ethyl-3-(3-dimethylaminopropyl)carbodiimide-A Critical Assessment. Bioconjug. Chem. 2008, 19, 1945-1950. [CrossRef] [PubMed]

13. Lin, Y.; Lu, F.; Tu, Y.; Ren, Z. Glucose Biosensors Based on Carbon Nanotube Nanoelectrode Ensembles. Nano Lett. 2004, 4, 191-195. [CrossRef]

14. Dudina, A.; Seichepine, F.; Chen, Y.; Stettler, A.; Hierlemann, A.; Frey, U. Monolithic CMOS sensor platform featuring an array of 9'216 carbon-nanotube-sensor elements and low-noise, wide-bandwidth and wide-dynamic-range readout circuitry. Sens. Actuators B Chem. 2019, 279, 255-266. [CrossRef] [PubMed] 
15. Rothe, J.; Frey, O.; Stettler, A.; Chen, Y.; Hierlemann, A. CMOS chip for electrochemical monitoring of the metabolic activity of biological cells. In Proceedings of the 2012 IEEE Sensors, Taipei, Taiwan, 28-31 October 2012; pp. 1-4. [CrossRef]

16. Grassi, M.; Malcovati, P.; Baschirotto, A. A 160 dB equivalent dynamic range auto-scaling interface for resistive gas sensors arrays. IEEE J. Solid-State Circuits 2007, 42, 518-527. [CrossRef]

17. Seichepine, F.; Rothe, J.; Dudina, A.; Hierlemann, A.; Frey, U. Dielectrophoresis-Assisted Integration of 1024 Carbon Nanotube Sensors into a CMOS Microsystem. Adv. Mater. 2017, 29, 1606852. [CrossRef] [PubMed]

18. Stern, E.; Wagner, R.; Sigworth, F.J.; Breaker, R.; Fahmy, T.M.; Reed, M.A.; Engineering, B.; Box, P.O.; Haven, N. Importance of the Debye Screening Length on Nanowire Field Effect Transistor Sensors. NIH Public Access. 2009, 7, 3405-3409. [CrossRef]

19. Schuvailo, O.M.; Soldatkin, O.O.; Lefebvre, A.; Cespuglio, R.; Soldatkin, A.P. Highly selective microbiosensors for in vivo measurement of glucose, lactate and glutamate. Anal. Chim. Acta 2006, 573-574, 110-116. [CrossRef]

20. Fields, R.D.; Stevens-Graham, B. Neuroscience: New insights into neuron-glia communication. Science 2002, 298, 556-562. [CrossRef]

21. Yoon, H.; Ko, S.; Jang, J. Field-effect-transistor sensor based on enzyme-functionalized polypyrrole nanotubes for glucose detection. J. Phys. Chem. B 2008, 112, 9992-9997. [CrossRef]

22. Besteman, K.; Lee, J.O.; Wiertz, F.G.M.; Heering, H.A.; Dekker, C. Enzyme-coated carbon nanotubes as single-molecule biosensors. Nano Lett. 2003, 3, 727-730. [CrossRef]

23. Star, A.; Gabriel, J.C.P.; Bradley, K.; Grüner, G. Electronic detection of specific protein binding using nanotube FET devices. Nano Lett. 2003, 3, 459-463. [CrossRef]

24. Sharf, T.; Kevek, J.W.; Deborde, T.; Wardini, J.L.; Minot, E.D. Origins of charge noise in carbon nanotube field-effect transistor biosensors. Nano Lett. 2012, 12, 6380-6384. [CrossRef]

25. Lee, B.Y.; Seo, S.M.; Lee, D.J.; Lee, M.; Lee, J.; Cheon, J.-H.; Cho, E.; Lee, H.; Chung, I.-Y.; Park, Y.J.; et al. Biosensor system-on-a-chip including CMOS-based signal processing circuits and 64 carbon nanotube-based sensors for the detection of a neurotransmitter. Lab Chip 2010, 10, 894-898. [CrossRef] [PubMed]

(C) 2019 by the authors. Licensee MDPI, Basel, Switzerland. This article is an open access article distributed under the terms and conditions of the Creative Commons Attribution (CC BY) license (http://creativecommons.org/licenses/by/4.0/). 